\title{
Entrainment and mixed layer dynamics of a surface-stress-driven stratified fluid
}

\author{
G.E. MANUCHARYA $\mathrm{N}^{1} \dagger$ and C.P. CAULFIEL D ${ }^{2,3}$ \\ ${ }^{1}$ Woods Hole Oceanographic Institution, Woods Hole, MA \\ ${ }^{2}$ BP Institute, University of Cambridge, Madingley Road, Cambridge CB3 0EZ, UK \\ ${ }^{3}$ Department of Applied Mathematics and Theoretical Physics, University of Cambridge, \\ Centre for Mathematical Sciences, Wilberforce Road, Cambridge CB3 0WA, UK
}

(Received 12 December 2014)

We consider experimentally a linearly stratified fluid with buoyancy frequency $N$ in a cylinder subject to surface-stress forcing from a disc of radius $R$ spinning at a constant angular velocity $\Omega$. We observe the growth of the disc-adjacent turbulent mixed layer bounded by a sharp primary interface with a constant thickness $l_{I}$. To a good approximation the depth of the mixed layer scales as $h_{U} / R \sim(N / \Omega)^{-2 / 3}(\Omega t)^{2 / 9}$. Generalising the previous arguments and observations of Shravat, Cenedese \& Caulfield (2012), we show that such a deepening rate is consistent with three central assumptions that allow us to develop a phenomenological energy balance model for the entrainment dynamics. First, the total kinetic energy of the deepening mixed layer $\mathcal{K}_{U} \propto h_{U} u_{U}^{2}$, where $u_{U}$ is a characteristic velocity scale of the turbulent motions within the upper layer, is essentially independent of time and the buoyancy frequency $N$. Second, the scaled entrainment parameter $E=\dot{h}_{U} / u_{U}$, depends only on the local interfacial Richardson number $R i_{I}=\left(N^{2} h_{U} l_{I}\right) /\left(2 u_{U}^{2}\right)$. Third, the potential energy production is driven by the local energy input at the interface, and hence is proportional to the third power of this characteristic velocity $u_{U}$. We establish that internal consistency between these assumptions implies that the rate of increase of the potential energy decreases with $R i_{I}$, suggesting following Phillips (1972), that the mixing in the vicinity of the primary interface leads to the spontaneous appearance of secondary partially-mixed layers which we indeed observe experimentally below the primary interface.

\section{Introduction}

The interaction between shear-driven turbulence and density stratification is a key process in a wide array of geophysically relevant flows, and the ensuing vertical mixing is of central importance in understanding the flow of the world oceans (see Wunsch \& Ferrari 2004; Ivey, Winters \& Koseff 2008; Ferrari \& Wunsch 2009, for reviews). The dynamics of this interaction, in particular the interconnected energetics of turbulent dissipation, larger scale 'stirring' and smaller-scale irreversible mixing, is extremely complex and subtle. There has been a large amount of research activity attempting to parameterise irreversible mixing in terms of larger scale bulk measures of the flow (e.g. Linden 1979; Fernando 1991; Ivey, Winters \& Koseff 2008).

Of central and ongoing interest are two inter-related questions. First, how is the kinetic energy, injected at some relatively large scale, apportioned between irreversible mixing, leading to an increase in the gravitational potential energy, and turbulent viscous dissipation; i.e. how 'efficient' is the mixing? Second, can the mixing in stratified flows be characterised by some relatively large-scale overturning process that tends to smear out

$\dagger$ Email address for correspondence: gmanucharyan@whoi.edu 
density gradients, and hence can be considered as a diffusive process, or is it more appropriately characterised as a 'scouring' process that tends to maintain or indeed sharpen density gradients within a flow? These two questions are inter-related, since as originally argued by Phillips (1972), if the rate of increase of potential energy decreases with increasing stratification there will be a tendency of the flow to form 'layers' of relatively well-mixed fluid separated by thinner 'interfaces' of substantially stronger gradients.

Recent experimental studies of stratified Taylor-Couette flow, i.e. an annular stratified flow driven by rotation of the inner cylindrical boundary (see Woods et al. (2010); Oglethorpe, Caulfield \& Woods (2013)) suggest strongly that non-diffusive 'scouring' entrainment processes do occur, supporting a large body of previous experimental work, dating back at least to Turner (1968) and Kato \& Phillips (1969). Quite recently, Oglethorpe, Caulfield \& Woods (2013) established in stratified Taylor-Couette flow that the mixing does indeed vary non-monotonically with stratification, and that layers do indeed spontaneously form in an initially linearly stratified fluid. It is clearly of interest to build upon such studies to establish whether anything generic can be stated about the mixing processes that occur within stratified turbulent flows, and so it is natural to consider a range of flow geometries and forcing mechanisms.

Of specific interest, as it is perhaps more characteristic of real geophysical situations than the Taylor-Couette situation, is mixing driven primarily by vertical shear. In this case the driving mechanism in the velocity field is in direct opposition to the stabilising mechanism of the static stability. A particularly appropriate experimental flow geometry, closely related to the flow geometry originally considered by Kato \& Phillips (1969), is that of a stratified fluid in a cylindrical tank driven by a disc of radius $R$ (close to the radius of the cylinder) rotating at a constant angular rotation rate $\Omega$ at one of the boundaries. Boyer et al. (1997) and Davies et al. (1995) considered two-layer and initially linearly stratified fluid respectively driven by a disc just above the base of the cylinder, and observed that a well-mixed layer developed in the vicinity of the disc whose depth appeared to deepen linearly with time.

Both Boyer et al. (1997) and Davies et al. (1995) argued that the potential energy production due to the local entrainment can be related linearly to the power supplied at the interface by the mobilised upper layer. In turn, the power input is proportional to the third power of some characteristic velocity $u_{U}^{3}$. They further argued that this characteristic velocity should be constant in time $u_{U} \propto \Omega R$ crucially independent of the present depth of the mixed layer $h_{U}$. Therefore, the combination of these two arguments implied that the rate of increase of potential energy was constant in time.

In the initially two-layer case, with initial densities $\rho_{U}(0)$ and $\rho_{L}>\rho_{U}(0)$ and initial upper layer depth $h_{U}(0)$, conservation of mass implies straightforwardly that $g_{U}^{\prime} h_{U}=$ $g\left(\rho_{L}-\rho_{U}(t)\right) / \rho_{0}=C_{M}$, a constant in time, where $\rho_{0}$ is some reference density. Furthermore, Boyer et al. (1997) showed that an appropriately defined potential energy of the upper layer is proportional to $g_{U}^{\prime} h_{U}^{2}=C_{M} h_{U}$, and thus that the rate of change of potential energy was directly proportional to $d h_{U} / d t$. Therefore, they predicted that depth of the well-mixed layer should deepen linearly in time for an initially two-layer stratification, a prediction which was supported by admittedly sparse experimental evidence. On the other hand, for the initially linearly stratified case with constant buoyancy frequency $N$, as we discuss in more detail in section 3, the potential energy of the upper layer is proportional to $N^{2} h_{U}^{3}$. Since they argued that the rate of increase with time of this quantity should be constant, Davies et al. (1995) predicted that $h_{U} \propto(\Omega t)^{1 / 3}$, and they did indeed observe a decrease in the rate of change of the depth of the upper mixed layer, which is consistent with this argument, though once again there were relatively few measurements of density profiles reported. 
Shravat, Cenedese \& Caulfield (2012) (henceforth referred to as SCC12) considered a similar two-layer flow to Boyer et al. (1997), focussing on the entrainment, mixing and homogenization of an initially two-layer stratification in a cylindrical tank, driven by a disc rotating with constant angular frequency $\Omega$ now at the upper surface of the fluid. They observed that the depth $h_{U}(t)$ of the upper mixed layer did not increase linearly with time, unlike the predictions of Boyer et al. (1997), but rather $h_{U} / R \sim(1+C \Omega t)^{2 / 5}$, for some empirical constant $C$. They showed that this scaling was consistent with a model based on the concept that the power injected into the flow by the rotating disc could not maintain constant characteristic velocities in the upper layer for sufficiently deep upper layers because this would demand an ever-increasing power both to mobilise the deepening fluid and to account for the ever-increasing viscous dissipation. Indeed, they demonstrated that the experimentally measured rate of deepening was consistent with the assumption that the kinetic energy $\mathcal{K}_{U}$ in the upper layer, defined as

$$
\mathcal{K}_{U}=\frac{1}{2} \int_{0}^{h_{U}} \int_{0}^{2 \pi} \int_{0}^{R} \rho|\mathbf{u}(r, \theta, z, t)|^{2} r d r d \theta d z=\frac{1}{2} \rho_{L} \pi R^{2} h_{U} u_{U}^{2},
$$

tended to a constant in time, thus making it possible to relate $u_{U}$ to $h_{U}^{-1 / 2}$. Central to their argument was the concept that 'mixing' should be considered as a two stage process. The local 'entrainment' across the interface is driven by a turbulent stress due to the mobilised upper layer, which is then followed by a 'homogenisation' of this entrained fluid throughout the turbulent deepening upper mixed layer.

Using this inherently local entrainment model, they were able to construct a selfconsistent model for deepening of the mixed layer, recovering the $(\Omega t)^{2 / 5}$ scaling. This model, which they referred to as a constant-disc-power or ' $\mathrm{P}$ ' model, is still based around the assumption that the characteristic turbulent velocity scale important for entrainment at the interface is proportional to the characteristic velocity scale $u_{U}$ in the upper layer, but crucially allows for this scale to vary (and indeed decrease) with time. Considering the global energy budget of the well-mixed layer required for mobilisation and homogenisation, they argued that a constant kinetic energy $\mathcal{K}_{U}$ required a balance between power injected by the rotating disc, the turbulent dissipation of the entire layer, and the total power demand of both the local entrainment and the homogenisation of the fluid density throughout the upper layer. However, the constant kinetic energy scaling only emerges at later time, or equivalently for sufficiently deep upper layers, and so there were some technical difficulties in being able to run experiments of sufficiently long duration.

Furthermore, and more significantly, these two-layer experiments could shed no light on the tendency of the stratified mixing to lead to layering (or not). The conductivity probe density measurements demonstrated that the primary interface between the wellmixed upper layer and the largely quiescent lower layer had a characteristic thickness scale $l_{I} \sim O(1 \mathrm{~cm})$ which did not vary measurably with time. Therefore, under the hypothesis that $\mathcal{K}_{U}$ remains constant with time, the interfacial Richardson number $R i_{I}$, is itself a constant with time:

$$
R i_{I}=\frac{g_{U}^{\prime} l_{I}}{u_{U}^{2}}=\frac{g_{U}^{\prime} h_{U} l_{I}}{h_{U} u_{U}^{2}}=C_{R i}
$$

since as already noted by Boyer et al. (1997), $g_{U}^{\prime} h_{U}=C_{M}$, a constant. This constraint unfortunately makes it impossible to determine how the entrainment local to the interface depends on $R i_{I}$ - a key to determining whether secondary layer formation is to be expected via the Phillips mechanism.

In light of these observations, in this paper we consider the different flow initial condition in the same geometry as SCC12, that of an initially linearly stratified fluid as 
considered by Davies et al. (1995), so that we can address two primary objectives. Firstly, we wish to verify if, analogously to the situation considered in SCC12, the aasumption that the kinetic energy of the upper layer tends towards a constant, and hence that $u_{U} \propto h_{U}^{-1 / 2}$ leads to a better prediction for the evolution of the mixed layer depth than the assumption of Davies et al. (1995) that $u_{U} \propto \Omega R$. Secondly, we wish to explore how the local entrainment varies with the interfacial stratification, and if its variation is such that we expect layer development by the Phillips mechanism. It is important to stress that we are interested in the bulk properties of the flow at sufficiently late time, rather than the initial spin-up instabilities of the flow or the specific character of the flow in the mixed layer (see Davies et al. (1995), Munro \& Davies (2006) and Munro, Foster \& Davies (2010) for consideration of these flow dynamics). In particular, we are interested in layers which develop and persist at 'late' times below the primary interface due to its particular mixing dynamics, rather than larger-scale, radially spreading intruding layers as observed by Davies et al. (1995).

To consider these two issues, the rest of the paper is organised as follows. In section 2 , we describe our experimental procedure and present our observational results. By measuring large numbers of density profiles, we demonstrate that the mixed layer deepens at a rate slower than that predicted by Davies et al. (1995), thus suggesting that the assumption that $u_{U} \propto \Omega R$ is not appropriate for all times. Also we show that secondary layering is regularly observed, strongly suggesting that the Phillips mechanism may be occuring. In section 3, we generalise the constant-disc-power ' $\mathrm{P}$ ' model presented in SCC12 with the aim of explaining the experimental findings. We demonstrate that our model is consistent with the observed dependence of the depth of the mixed layer on both time and the external parameters $\Omega$ and $N$, and also that the rate of increase of potential energy is a decreasing function of $R i_{I}$, consistently with the observations of robust secondary layers developing below the primary interface. Finally, in section 4, we draw our conclusions.

\section{Experiments}

\subsection{Experimental procedure}

We have conducted a series of experiments on a stratified fluid in a cylindrical tank forced at the surface by a rotating horizontal disc, using the same experimental equipment as described in SCC12. We fill a cylindrical tank $(30 \mathrm{~cm}$ height and $30 \mathrm{~cm}$ diameter) with fluid of a consistent height $H=27 \mathrm{~cm}$. Using the conventional Oster double-bucket technique, we set an initial linear density stratification relative difference between the density at the top of the fluid $\rho_{T}$ and the bottom $\rho_{B}$ of the fluid $\left(\rho_{B}-\rho_{T}\right) / \rho_{T} \in(0.02,0.2)$. We measure vertical density profiles using a conductivity probe moving vertically downwards with a speed of $3 \mathrm{~mm} \mathrm{~s}^{-1}$ at a sampling rate taking 100 measurements every centimetre. The profiled depth of the fluid is $20 \mathrm{~cm}$, excluding regions close to the disc and to the bottom of the tank. We convert conductivity measurements into density using a calibration based around a third degree polynomial. All fluid is at the (controlled) room temperature of $20^{\circ} \pm 0.5^{\circ} \mathrm{C}$. Therefore, the Schmidt number for the salt stratified water is nearly constant $S c=\nu / \kappa \sim 700$, where $\nu$ is the kinematic viscosity, and $\kappa$ is the salt diffusivity.

Following SCC12, we drive a horizontal disc (radius $R=12 \mathrm{~cm}$ ) in contact with the surface of the fluid with constant angular rotation rate $\Omega$. We keep the aspect ratio $R / H$ fixed for all experiments. Thus, there are two natural control parameters for the experiments: the rotation rate $\Omega$ and the buoyancy frequency $N$ defined by the initial 


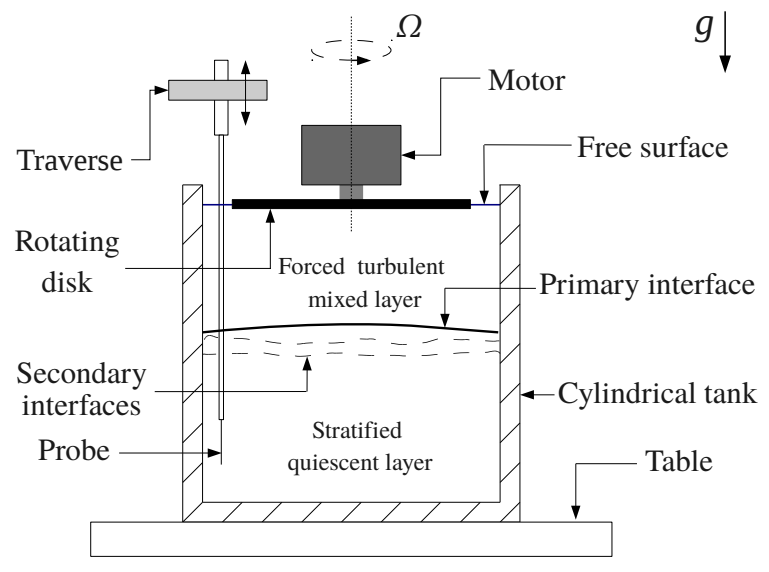

Figure 1: A schematic of the experimental set-up showing a cylindrical tank filled with stratified fluid, forced at the surface by a rotating disc, and measured by a traversing conductivity probe from $z=0$ (the upper surface) to $z=-H$ (the tank base).

linear stratification

$$
\rho_{L}(z)=\rho_{T}\left(1-\frac{N^{2}}{g} z\right) ; N^{2}=\frac{g\left(\rho_{B}-\rho_{T}\right)}{\rho_{T} H},
$$

defining vertical coordinate $z$ increasing upwards from $z=-H$ at the base of the tank to $z=0$ at the disc. The ratio of $N$ and $\Omega$ define a natural global nondimensional parameter for the 'profile' Richardson number $R i_{P}$ defined as

$$
R i_{P}=N^{2} / \Omega^{2} .
$$

We observe that the entire column of fluid in the tank becomes well mixed over a mixing time $T_{M} \in(1.2-72) \times 10^{3} \mathrm{~s}$. As the characteristic flow speeds (and hence the intensity of the turbulence leading to stratified mixing) depend on the disc rotation rate $\Omega$, it is natural to nondimensionalize time $t$ as $\hat{t}=\Omega t$. We have analysed 20 experiments, with $0.5 \mathrm{~s}^{-1} \leqslant N \leqslant 1.72 \mathrm{~s}^{-1}$ and $0.72 \mathrm{rad} \mathrm{s}^{-1} \leqslant \Omega \leqslant 3.14 \mathrm{rad} \mathrm{s}^{-1}$, resulting in $0.14 \leqslant$ $R i_{P} \leqslant 2.25$ (as listed in table 1 ).

\subsection{Qualitative observations}

As discussed in more detail in SCC12, the characteristic Reynolds number $R e=\Omega R^{2} / \nu \sim$ $O\left(10^{5}\right)$, and so a turbulent well-mixed mobilised layer develops near the disc, with almost homogeneous density distribution. Although there is undoubtedly a large-scale radial flow (see Davies et al. (1995), Munro \& Davies (2006), Munro, Foster \& Davies (2010) for more details), we are principally interested in the bulk later-time properties of the deepening layer. As discussed in SCC12, there is a power demand for this deepening, as the gravitational potential energy of the fluid increases as the layer deepens, since entrainment and homogenisation processes effectively lift the centre of mass of the water column by transporting dense fluid upward in the gravitational field. Furthermore, an increasingly strong, and noticeably sharp density jump forms at the base of the mixed layer which effectively shields the quiescent layer underneath it from penetration and overturning by turbulent eddies. 


\begin{tabular}{ccccc|ccccc}
\hline$R i_{P}=N^{2} / \Omega^{2}$ & $N$ & $\Omega$ & $\alpha$ & $A$ & $R i_{P}=N^{2} / \Omega^{2}$ & $N$ & $\Omega$ & $\alpha$ & $A$ \\
\hline 0.14 & 0.5 & 1.34 & 0.18 & 1.17 & 0.95 & 1.3 & 1.34 & 0.24 & 0.54 \\
0.16 & 0.84 & 2.08 & 0.21 & 1.07 & 1 & 1.7 & 1.7 & 0.225 & 0.51 \\
0.2 & 0.5 & 1.13 & 0.2 & 0.98 & 1.05 & 0.98 & 0.95 & 0.24 & 0.56 \\
0.3 & 1.72 & 3.14 & 0.18 & 0.96 & 1.09 & 1.18 & 1.13 & 0.25 & 0.49 \\
0.4 & 0.94 & 1.5 & 0.23 & 0.67 & 1.18 & 0.78 & 0.72 & 0.24 & 0.53 \\
0.43 & 1.12 & 1.7 & 0.2 & 0.84 & 1.57 & 1.42 & 1.13 & 0.23 & 0.51 \\
0.52 & 0.72 & 0.52 & 0.2 & 0.75 & 1.6 & 1.5 & 1.18 & 0.24 & 0.51 \\
0.54 & 1.1 & 1.5 & 0.22 & 0.68 & 1.67 & 1.46 & 1.13 & 0.25 & 0.42 \\
0.65 & 1.37 & 1.7 & 0.23 & 0.63 & 1.87 & 1.3 & 0.95 & 0.246 & 0.44 \\
0.67 & 1.6 & 1.94 & 0.22 & 0.68 & 2.25 & 1.7 & 1.13 & 0.225 & 0.56 \\
\hline
\end{tabular}

Table 1: Table of experimental parameters for the 20 conducted experiments: The last two columns show the coefficients of the power law fit for evolution of the upper mixed layer depth $h_{U} / R=A(\Omega t)^{\alpha}$, where $t$ is the time from the start of the experiment.

A mean azimuthal flow clearly develops due to the coherent rotation of the disc, thus creating a shear across the interface between the mobilised upper layer and the quiescent lower layer. We observe shear-driven overturning behaviour, relatively early in an experiment when the mixed layer is relatively shallow, particularly for experiments with relatively small values of $R i_{P}$, where it is reasonable to suppose that the stratification is 'weak' at the interface.

However, as mentioned in the introduction, particularly when the density jump across the interface is 'strong' in some sense, as the turbulent eddies approach the interface they 'scour' dense fluid into the upper layer, and so maintain 'sharp' density gradients in the vicinity of the interface. Such non-diffusive scouring dynamics tended to be observed when the upper layer was sufficiently deep, particularly for larger values of $R i_{P}$.

Both diffuse primary interfaces associated with overturning, and sharper interfaces associated with scouring can be observed in the normalised density profiles $\hat{\rho}$, defined as

$$
\hat{\rho}=\frac{\rho-\rho_{T}}{\rho_{B}-\rho_{T}},
$$

and measured with the conductivity probe. Such profiles (offset by 0.04 for clarity) are plotted in figure $2 \mathrm{a}$ for an experiment with $R i_{P}=0.67$. The upper layer is clearly very well mixed, although consistently with the observations of SCC12 and Davies et al. (1995), the depth of the mixed layer does not increase linearly with time, but rather clearly descends at a decreasing rate with time. (The unphysical offset in the top few centimetres of the profile is associated with an oscillation in the traverse when it starts to move.)

In figure $2 \mathrm{~b}$, for the same experiment we plot the time evolution of the (natural) logarithm of the buoyancy frequency $N$ in the $3 \mathrm{~cm}$ below the (moving) primary interface located at $z=-h_{U}$. We identify the primary interface location $-h_{U}$ as the global (in space) maximum of the buoyancy frequency; this appears to be a robust way to identify $h_{U}$ without ambiguity. At earlier times, there is clear evidence of unstable stratification, resulting from the overturning of energetic shear-driven eddies at the base of the mixed layer. Conversely, at later times, there is both a sharper primary density interface, and 

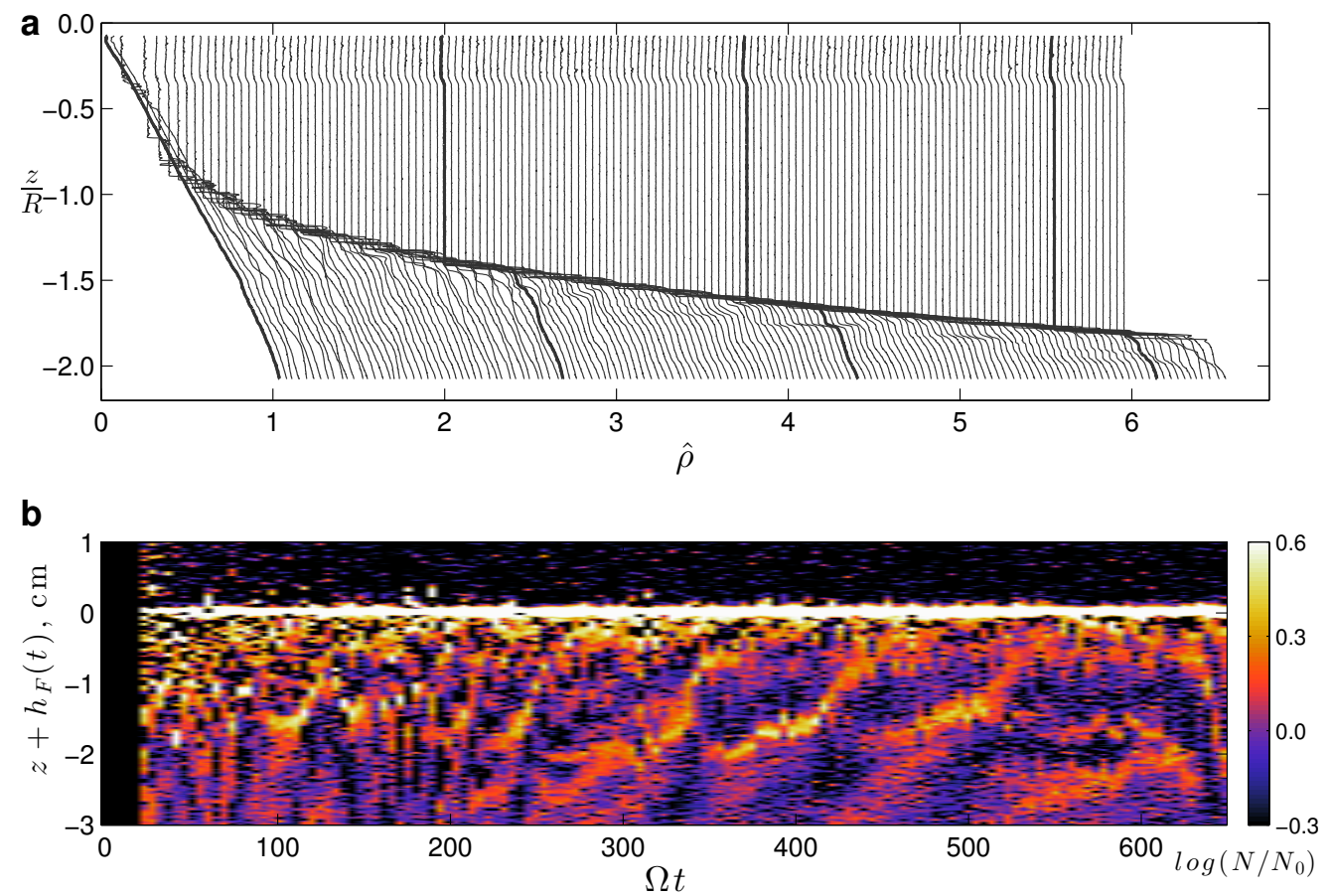

Figure 2: a: Sequence of profiles of normalised density $\hat{\rho}$ as defined in (2.3) separated in time by intervals of $\Omega \Delta t=4.6\left(R i_{P}=0.67\right)$. Consecutive profiles are offset in the $x$-coordinate by 0.04 from each other. b: Spatio-temporal variation of $\log N$ for the same experiment in the $3 \mathrm{~cm}$ below the primary interface.

also long-lived secondary interfaces below the primary interface, although they exhibit complex long-time-scale dynamics, in particular through intermittent disappearance and reappearance. We believe that these interfaces form below the primary interface, and do not appear to be associated with the radial collapse of regions of partially mixed fluid above the primary interface, which always appears to be well mixed.

The primary interfacial thickness is both sharp $l_{I} \sim O(0.1-1 \mathrm{~cm})$ and constant in time, consistently with the observations of SCC12. Our observations support the hypothesis that the entrainment process at the interface over much of the flow evolution may be characterised as a 'scouring' process, that continually maintains a relatively sharp gradient at the interface between the two primary layers.

\subsection{Quantitative analysis}

Motivated by the arguments of Davies et al. (1995) and SCC12, we postulate that

$$
\hat{h}_{U}=h_{U} / R=A\left(R i_{P}\right) \hat{t}^{\alpha\left(R i_{P}\right)},
$$

where the premultiplying scaling factor $A\left(R i_{P}\right)$ and the power law $\alpha\left(R i_{P}\right)$ are allowed in principle to be functions of $R i_{P}$. In figure $3 \mathrm{a}$, we plot the nondimensionalised mixed layer depth $\hat{h}_{U}$ against nondimensional time $\hat{t}$ for three different typical values of $R i_{P}=0.3$, 0.52 and 1.09. A power-law approximation appears to fit each dataset well, as plotted with a solid line using the values for $\alpha\left(R i_{P}\right)$ and $A\left(R i_{P}\right)$ listed in table 1 . Two aspects are immediately apparent. First, in each case, the power law dependence is close to $\alpha \simeq 2 / 9$, as shown in the inset. Indeed, as shown in the table and figure $3 \mathrm{~b}$, there is 

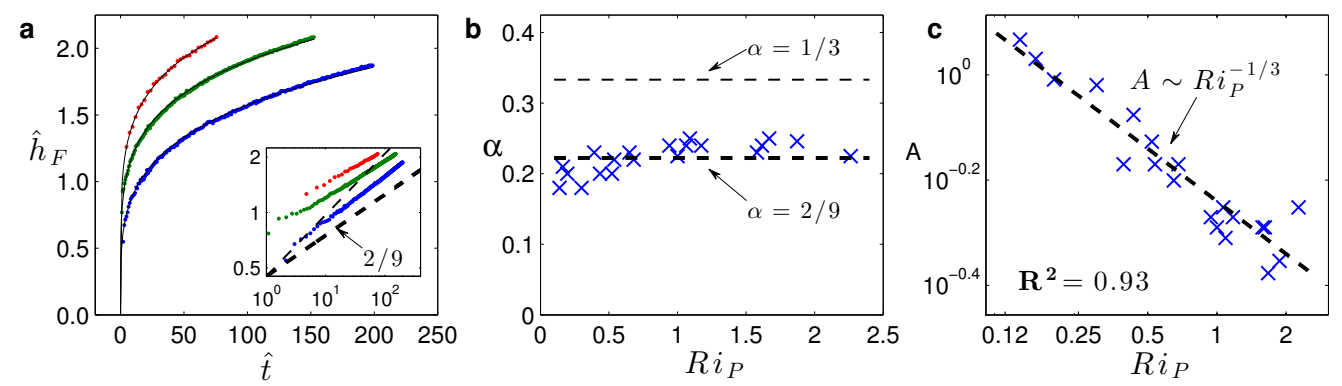

Figure 3: a) Time evolution of the nondimensional mixed layer depth $\hat{h}$ for three different experiments: $R i_{P}=0.3$ (red dots), 0.52 (green), and 1.09 (blue); inset shows the same data on a log-log plot. b) Best-fit values of the power-law coefficient $\alpha$ (defined in 2.4) plotted as a function of $R i_{P}$ for different experiments (crosses). The lines $\alpha=2 / 9$ and $\alpha=1 / 3$ are plotted with dashed lines. c) Log-log plot of the best-fit values of the scaling factor $A\left(R i_{P}\right)$ (as defined in (2.4)) (crosses). The best fit line $A=0.57 R i_{P}^{-1 / 3}$ is plotted with a dashed line. Numerical values of $\alpha, A$, and $R i_{P}$ are listed in table 1.

weak dependence of the power law coefficient $\alpha$ on $R i_{P}$ : the mean value for all twenty experiments (as shown with a dashed line in figure $3 \mathrm{~b}$ ) is $\alpha=0.229 \simeq 2 / 9$ with a standard deviation of 0.02. The data is not consistent with the prediction of Davies et al. (1995) that $\alpha=1 / 3$. Secondly, the depth of the mixed layer increases more rapidly as $R i_{P}$ decreases: $A\left(R i_{P}\right) \simeq A_{c} R i_{P}^{-1 / 3}$, where $A_{c}=0.57$, with $\mathbf{R}^{2}=0.93$ (figure $3 \mathrm{c}$ ).

Unlike the observations of SCC12, there is no apparent initial time offset in the data for the power law dependence, This observation is perhaps a little surprising, since, as discussed above, the physical mechanisms of entrainment and mixing appear to change during the flow evolution from at least partially shear-driven overturning to scouring driven by impinging turbulent eddies. However, since the experimental evidence is that $\alpha<1 / 3$, it appears appropriate to relax the central assumption of Davies et al. (1995), that the characteristic velocity of the upper layer $u_{U}$ does not vary with time. Therefore, we investigate whether some generalisation of the constant-disc-power ' $\mathrm{P}$ ' model discussed in SCC12 (based around the assumption that the kinetic energy of the upper layer tends towards a constant and so $u_{U} \propto h_{U}^{-1 / 2}$ ) may prove to be successful in describing the observed dynamics of the upper mixed layer.

\section{Mixing model}

\subsection{Layer properties}

As a first step to develop a model for the evolution of the upper well-mixed layer, we define the reduced gravity at a given location $z<0$ in the stratified layer as

$$
g_{L}^{\prime}(z)=\frac{g\left(\rho-\rho_{T}\right)}{\rho_{T}}=-N^{2} z,
$$

using (2.1). When the upper mixed layer has depth $h_{U}$, the reduced gravity of the upper layer $g_{U}^{\prime}$, and hence the interfacial Richardson number $R i_{I}$ are given by

$$
g_{U}^{\prime}=\frac{1}{h_{U}} \int_{-h_{U}}^{0} g_{L}^{\prime} d z=\frac{N^{2} h_{U}}{2} ; R i_{I}=\frac{\left[g_{L}^{\prime}\left(-h_{U}\right)-g_{U}^{\prime}\right] l_{I}}{u_{U}^{2}}=\frac{N^{2} h_{U} l_{I}}{2 u_{U}^{2}},
$$


making the conventional assumptions that there is a characteristic velocity scale for the upper layer $u_{U}$, and that the interfacial thickness $l_{I}$ is constant in time.

Entraining fluid across the interface increases the density in the upper mixed layer and clearly increases the potential energy of the system $\mathcal{P}_{U}\left(h_{U}\right)$, which we define as

$$
\mathcal{P}_{U}\left(h_{U}\right)=\rho_{T} \pi R^{2} \int_{-h}^{0} \frac{g\left(\rho_{U}-\rho\right) z}{\rho_{T}} d z=\frac{\rho_{T} \pi R^{5} N^{2}}{12}\left(\frac{h_{U}^{3}}{R^{3}}\right)=\mathcal{P}_{S} \hat{h}_{U}^{3},
$$

also defining the characteristic scale $\mathcal{P}_{S}$. We now make an assumption, ' $\mathrm{K}$ ', that the total kinetic energy of the upper layer (1.1) may be considered to be a constant independent of time. This was a central assumption of SCC12 consistent with their experimental data. In this flow, we assume further that this constant value of kinetic energy does not depend on the buoyancy frequency of the lower layer, and so

$$
\mathcal{K}_{U}=\frac{1}{2} \rho_{T} \pi R^{2} h_{U} u_{U}^{2}=\left(\frac{1}{2} \rho_{T} \pi R^{2}\right) C_{K} \Omega^{2} R^{3},
$$

for some empirical constant $C_{K}$, using the Boussinesq approximation.

The key argument is that the velocity in the upper layer is fundamentally set by a balance between the forcing of the disc and the turbulent dissipation and homogenisation in the layer, and the particular properties of the lower quiescent layer play a much less significant role. Using assumption ' $\mathrm{K}$ ', it is then possible to relate $u_{U}$ to $h_{U}$ :

$$
u_{U}=\Omega R\left(\frac{C_{K}}{\hat{h}_{U}}\right)^{1 / 2},
$$

crucially showing that we are assuming that $u_{U}$ decreases as the mixed layer deepens.

\subsection{Time dependence of $\hat{h}_{u}$}

We now make a second assumption, 'P', that the potential energy of the system increases due to entrainment at the interface at a rate given by a fixed (empirically determined) proportion $\lambda$ of the power $\mathcal{P}_{I}$ supplied at the interface by the interfacial stress $\sigma$,

$$
\mathcal{P}_{I}=\frac{d}{d t} \mathcal{W}=\pi R^{2} u_{I} \sigma=\pi R^{2} c_{D} \rho_{T} u_{U}^{3},
$$

following SCC12, and using the Boussinesq assumption, where $c_{D}$ is some empirically determined drag coefficient, and $\mathcal{W}$ is the work done locally at the interface by the disc forcing via the action of the turbulent flow. Therefore, using (3.3), (3.5) and (3.6) assumption ' $\mathrm{P}$ ' yields a straightforward differential equation for $h_{U}$ which can be solved using the initial condition that $\hat{h}_{U}(0)=0$ :

$$
\frac{d}{d t}\left[\rho_{T} \pi R^{2} \frac{N^{2} h_{U}^{3}}{12}\right]=\lambda c_{D} u_{U}^{3}\left(\rho_{T} \pi R^{2}\right) \rightarrow \hat{h}_{U}=C_{K}^{3 / 2}\left(18 \phi\left[\frac{\Omega^{2}}{N^{2}}\right] \hat{t}\right)^{2 / 9},
$$

defining $\phi=\lambda c_{D}$. It is plausible that $\phi$ depends on some nondimensional function of $\Omega$ and $N$, associated with some dependence of the entrainment process on the developing stratification. We attempt to capture this dependence by making a third assumption, 'E', that the scaled entrainment parameter $E$, defined appropriately for this flow as

$$
E=\dot{h}_{U} / u_{u}
$$

(see SCC12 for further discussion) depends only on the present value of the interfacial Richardson $R i_{I}$, as defined in (3.2). Using (3.5), (3.7).

$$
E=\frac{4 \phi u_{U}^{2}}{N^{2} h_{U}^{2}}=\frac{4 \phi C_{K} \Omega^{2} R^{3}}{N^{2} h_{U}^{3}}=\phi^{1 / 3}\left(\frac{2}{3}\right)^{4 / 3}\left(\frac{\Omega}{N}\right)^{2 / 3} \hat{t}^{-2 / 3},
$$




$$
R i_{I}=\frac{N^{2} h_{U} l_{I}}{2 u_{U}^{2}}=\frac{N^{2} h_{U}^{2} l_{I}}{2 h_{U} u_{U}^{2}}=\phi^{4 / 9}\left(\frac{l_{I}}{2 R}\right) \frac{18^{4 / 9}}{C_{K}^{1 / 3}}\left(\frac{\Omega}{N}\right)^{-10 / 9} \hat{t}^{4 / 9} .
$$

Therefore, for assumption ' $\mathrm{E}$ ' to be valid for all time, the time dependence of $(3.9 a)$ and $(3.9 b)$ must be matched, which is equivalent to assuming that

$$
E=A_{R} R i_{I}^{-3 / 2}
$$

where $A_{R}$ is once again an empirical constant, and consistently with the previous observations of Kit, Berent \& Vajda (1980) in a flow with similar forcing. Since both $E$ and $R i_{I}$ both also depend on $\phi$ and $\Omega / N$ (and hence $R i_{P}$ ), this required power law dependence (3.10) also implies a consistency condition for $\phi$ :

$$
\phi=\frac{A_{R} C_{K}^{1 / 2}}{4}\left(\frac{l_{I}}{2 R}\right)^{-3 / 2} \frac{\Omega}{N}=\frac{A_{R} C_{K}^{1 / 2}}{4}\left(\frac{l_{I}}{2 R}\right)^{-3 / 2} R i_{P}^{-1 / 2} .
$$

Substituting this expression into (3.7), we obtain the final model prediction for the mixed layer depth $\hat{h}_{U}$ using the three key assumptions ' $\mathrm{K}$ ', 'P', and ' $\mathrm{E}$ ':

$$
\hat{h}_{U}=\left(\frac{9}{2} A_{R} C_{K}^{2}\right)^{2 / 9}\left(\frac{2 R}{l_{I}}\right)^{1 / 3} R i_{P}^{-1 / 3} \hat{t}^{2 / 9}=A_{c} R i_{P}^{-1 / 3} \hat{t}^{2 / 9} .
$$

This expression recovers precisely the observed scaling for $\hat{h}_{U}$ as shown in figure 3 .

\subsection{Implications}

Combining (3.9b) with (3.11), the interfacial Richardson number $R i_{I}$ can be expresses as a function of time and $R i_{P}$ as defined in $(2.2)$ :

$$
R i_{I}=\left(\frac{9 A_{R}}{2}\right)^{4 / 9}\left(\frac{l_{I}}{2 R}\right)^{1 / 3} R i_{P}^{1 / 3} \hat{t}^{4 / 9} .
$$

Since we now have derived scaling expressions for $\phi, u_{U}$ and $R i_{I}$ (and in particular $h_{U}$, $u_{U}$ and $R i_{I}$ are all functions of $\hat{t}$ ) it is thus possible to re-express the rate of change of the flow's potential energy $\mathcal{P}_{U}$ using (3.3) in terms of $R i_{I}$ and $R i_{P}$ :

$$
\frac{d}{d \hat{t}}\left(\frac{\mathcal{P}_{U}}{\mathcal{P}_{S}}\right)=\left(3 A_{R} C_{K}^{5 / 4}\right)\left(\frac{2 R}{l_{I}}\right)^{3 / 4} R i_{P}^{-1 / 4} R i_{I}^{-3 / 4}=\left(\frac{A_{\mathcal{P}}}{R i_{P} R i_{I}^{3}}\right)^{1 / 4}
$$

defining a new empirical constant $A_{\mathcal{P}}$.

Therefore, within our model, the rate of increase of the potential energy is predicted to be a decreasing function of the interfacial Richardson number. Such a dependence is all that is required by the arguments presented by Phillips (1972), and so secondary layering would be expected to occur in our flow. This is consistent with our observations, as is apparent in figure 2, even though the modelling effort leading to (3.14) is built on a strong set of assumptions. Since the decreasing dependence of $d \mathcal{P}_{U} / d t$ on $R i_{I}$ is predicted to occur throughout the lifetime of an experiment, this particular flow geometry seems to be particularly conducive to the detailed study of the development of layers within a forced stratified turbulent flow, an issue to which we intend to return in due course.

\section{Conclusions}

In this paper we have described the results of a sequence of experiments designed to study turbulent entrainment, mixing and homogenisation in a shear-driven turbulent 
stratified flow. In particular, we have studied the increase in depth $h_{U}$ of a surfacestress-driven mixed layer in a cylindrical tank initially filled with linearly stratified fluid. We demonstrate that the rate of increase of mixed layer depth decreases measurably with time, and furthermore that the data closely agrees with the scaling $h_{U} \propto$ $(\Omega / N)^{-2 / 3}(\Omega t)^{2 / 9}$ for a wide range of values of $\Omega$ and $N$. A key physical observation is that the interfacial thickness $l_{I}$ between the upper well-mixed turbulent layer and the quiescent linearly stratified lower layer appears to be 'scoured' to be close to a constant value both with respect to time and $R i_{P}$ as defined in (2.2). This observation is consistent with much previous work in a range of stratified entrainment and mixing studies (see e.g. the recent work of Oglethorpe, Caulfield \& Woods (2013)).

We present physical and mathematical arguments to justify the observed scaling for $h_{U}$, and demonstrate that this scaling is consistent with three central assumptions, building on previous research. Assumption ' $\mathrm{P}$ ', that the rate of the potential energy increase is proportional to the power supplied through turbulent interfacial stresses (in turn proportional to $u_{U}^{3}$ ), builds on the arguments of Davies et al. (1995) and Boyer et al. (1997). However, the central, and robust observation that the depth $h_{U} \propto \hat{t}^{2 / 9}$ appears to require that $u_{U}$ must also vary with time, contrary to the models presented by Davies et al. (1995) and Boyer et al. (1997). Building on SCC12, we make assumption 'K', that the kinetic energy of the upper layer tends towards a constant in time, which constant depends on the rotation rate $\Omega$, but is not dependent on the stratification of the lower, quiescent layer. This scaling yields the 'correct' observed time-dependence of $h_{U}$.

However, the fact that the lower layer is linearly stratified enables us to go further in modelling the interfacial entrainment dynamics, and use the conventional assumption, (see for example the review of Fernando (1991)), which we refer to as assumption ' $\mathrm{E}$ ', that the dynamics of the entrainment depends only on the (present) value of the interfacial Richardson number $R i_{I}$, as defined in (3.2). Since this in turn depends on time, assumption 'E' effectively imposes a consistency condition between $h_{U}$ and $R i_{I}$. As we demonstrate above, this consistency condition implies that $h_{U} \propto R i_{P}^{-1 / 3}$, which is strongly supported by our experimental data.

Furthermore, and perhaps more interestingly, internal consistency between our model assumptions and the data also implies that the rate of increase of potential energy, associated with entrainment across the density interface, actually decreases with the interfacial Richardson number $R i_{I}$. Such an effective decrease in buoyancy flux with sufficiently 'strong' overall stratification points towards the natural development of secondary layers at the interface, due to the physical argument originally presented by Phillips (1972). Such layers have been observed to develop in many different experimental situations (see the early review of Linden (1979), and Park, Whitehead \& Gnanadeskian (1994), Holford \& Linden (1999), Oglethorpe, Caulfield \& Woods (2013)) as well as in phenomenological models (e.g. Balmforth, Llewellyn Smith \& Young (1998)) that attempted to theoretically rationalise the observed layering. This experimental geometry seems to be particularly well-suited to investigate the creation and maintenance of such dynamically generated and maintained layered density distributions. The continual deepening of the primary mixed layer inevitably requires the interface bounding a secondary 'mixed' layer to be reformed and reinforced in a new, and always deeper, region of the linearly stratified lower layer. This experimental geometry allows such layers and interfaces to be observed over long time-periods (see figure $2 \mathrm{~b}$ ), and so is an ideal test-bed to develop improvements in larger scale parameterisations of ocean mixing (see for example Large, McWilliams \& Doney (1994)) which capture flow dynamics exhibiting spontaneous hy- 
drodynamical layer formation due to the 'Phillips' mechanism. We intend to report on just such an investigation of secondary layer dynamics in due course.

The experiments were conducted during the 2010 Geophysical Fluid Dynamics Program at Woods Hole Oceanographic Institution. Financial support from the National Science Foundation, the Office of Naval Research and Woods Hole Oceanographic Institution is gratefully acknowledged, as well as valuable technical support from Anders Hansen. The research activity of C.P.C. is supported by EPSRC Programme Grant EP/K034529/1 entitled 'Mathematical Underpinnings of Stratified Turbulence.'

\section{REFERENCES}

Balmforth, N. J., Llewellyn Smith, S. G. \& Young, W. R. 1998 Dynamics of interfaces and layers in a stratified turbulent fluid.

Boyer, D. L., Davies, P. A. \& Guo, Y. 1997 Mixing of a two-layer stratified fluid by a rotating disc. Fluid Dyn. Res. 21, 381-401.

Davies P. A., Guo, Y., Boyer D. L. \& Folkard, A. M. 1995 The flow generated by the rotation of a horizontal disc in a stratified fluid. Fluid Dyn. Res. 17, 27-47.

Fernando, H. J. S. 1991 Turbulent mixing in stratified fluids. Annu. Rev. Fluid Mech. 23, 455-493.

FERRARI, R. \& Wunsch, C. 2009 Ocean circulation kinetic energy: reservoirs, sources, and sinks. Annu. Rev. Fluid Mech. 41, 253-282.

Holford, J. M. \& Linden, P. F. 1999 Turbulent mixing in a stratified fluid. Dyn. Atmos. Ocean. 30, 173-198.

Ivey, G. N., Winters, K. B. \& Koseff J. R. 2008 Density stratification, turbulence, but how much mixing? Annu. Rev. Fluid Mech. 40, 169-184.

KAto, H. \& Phillips, O. M. 1969 On the penetration of a turbulent layer into stratified fluid. J. Fluid Mech. 37, 643-655.

Kit, E., Berent, E. \& VAJdA, M. 1980 Vertical mixing induced by wind and a rotating screen in a stratified fluid in a channel. J. Hydraul. Res. 18, 35-57.

Large, W. G., McWilliams, J. C. \& Doney, S. C. 1994 Oceanic vertical mixing: A review and a model with a nonlocal boundary layer parameterization. Rev. Geophys. 32, 363-403.

Linden, P. F. 1979 Mixing in stratified fluids. Geophys. Astrophys. Fluid Dyn. 13, 3-23.

Munro, R. J. \& DAvies, P. A. 2006 The flow generated in a continuously stratified rotating fluid by the differential rotation of a plane horizontal disc. Fluid Dyn. Res. 38, 522-538.

Munro, R. J., Foster, M. R. \& Davies, P. A. 2010 Instabilities in the spin-up of a rotating, stratified fluid. Phys. Fluids 22, Art. Number 054108.

Oglethorpe, R. L. F., Caulfield, C. P. \& Woods, A. W. 2013 Spontaneous layering in stratified turbulent Taylor-Couette flow. J. Fluid Mech. 721, R3, 12 pages.

Park, Y.-G., Whitehead, J. A. \& Gnanadeskian, A. 1994 Turbulent mixing in stratified fluids: layer formation and energetics. J. Fluid Mech. 279, 279-311.

Phillips, O. M. 1972 Turbulence in a strongly stratified fluid - is it unstable? Deep Sea Res. 19, 79-81.

Shravat, A., Cenedese, C. \& Caulfield, C. P. 2012 Entrainment and mixing dynamics of surface-stress-driven stratified flow in a cylinder. J. Fluid Mech. 691, 498-517. Referred to herein as SCC12.

Spigel, R. H., Imberger, J. \& Rayner, K. N. 1986 Modelling the diurnal mixed layer. Limnol. Oceanogr. 31, 533-566.

Turner, J. S. 1968 The influence of molecular diffusivity on turbulent entrainment across a density interface. J. Fluid Mech. 33, 639-656.

Woods, A. W., Caulfield, C. P., Landel, J. R. \& Kuesters, A. 2010 Non-invasive turbulent mixing across a density interface in a turbulent Taylor-Couette flow. J. Fluid Mech. 663, 347-357.

Wunsch C. \& Ferrari, R. 2004 Vertical mixing, energy and the general circulation of the oceans. Annu. Rev. Fluid Mech. 36, 281-314. 